RFP-2664

February 17, 1978
RFP-2664

February 17, 1978

\title{
SUMMARY OF BERYLLIUM SPECIFICATIONS
}

Richard R. Corle

Materials Technology

GENERAL METALLURGY

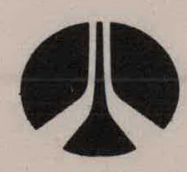

Rockwell International
Atomics International Division
Rocky Flats Plant
P.O. Box 464
Golden, Colorado 80401

U. S. DEPARTMENT OF ENERGY

CONTRACT EY-76-C-04-3533 


\section{LEGAL NOTICE}

This report was prepared as an account of work sponsored by the United States Government. Neither the United States nor the Department of Energy, nor any of their employees, nor any of their contractors, subcontractors, or their employees, makes any warranty, expressed or implied, or assumes any legal liability or responsibility for the accuracy, completenesss or usefulness of any information, apparatus, product or process disclosed, or represents that its use would not infringe privately owned rights.

Printed in the United States of America

Available from the

National Technical Information Service

U. S. Department of Commerce

Springfield, Virginia 22161

Price: Printed Copy $\$ 4.50$ Microfiche $\$ 3.00$

Price Is Subject to Change Without Notice 


\section{DISCLAIMER}

This report was prepared as an account of work sponsored by an agency of the United States Government. Neither the United States Government nor any agency Thereof, nor any of their employees, makes any warranty, express or implied, or assumes any legal liability or responsibility for the accuracy, completeness, or usefulness of any information, apparatus, product, or process disclosed, or represents that its use would not infringe privately owned rights. Reference herein to any specific commercial product, process, or service by trade name, trademark, manufacturer, or otherwise does not necessarily constitute or imply its endorsement, recommendation, or favoring by the United States Government or any agency thereof. The views and opinions of authors expressed herein do not necessarily state or reflect those of the United States Government or any agency thereof. 


\section{DISCLAIMER}

Portions of this document may be illegible in electronic image products. Images are produced from the best available original document. 
RFP-2664

February 17, 1978
RFP-2664

UC-25 MATERIALS

TID-4500-R66

\section{SUMMARY OF BERYLLIUM SPECIFICATIONS}

Richard R. Corle

Materials Technology

GENERAL METALLURGY
SUBJECT DESCRIPTORS

Beryllium Specifications

\section{ROCKWELL INTERNATIONAL ATOMICS INTERNATIONAL DIVISION ROCKY FLATS PLANT P. O. DOX 464 COLDEN, COLORADO 80401}

Prepared under Contract EY-76-C-04-3533 for the Albuquerque Operations Office U.S. Department of Energy

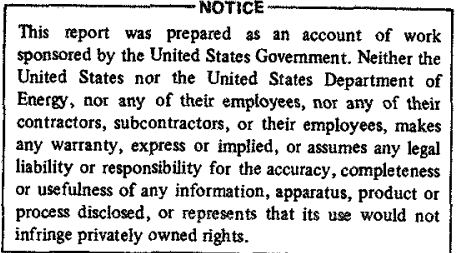

This wepared as an account of work Energy, nor any of their emplayees, nor any of their contractors, subcontractors, or their employees, makes liability or responsibility for the accuracy, completeness or usefulness of any information, apparatus, product or
process disclosed, or represents that its uae would not infringe privately owned rights. 
RFP-2664 


\title{
ACKNOWLEDGMENTS
}

\begin{abstract}
Appreciation is expressed to the following for their contributions: Stanley Beitscher from Research and Engineering for recommending the need for a beryllium specification summary and William W. Leslie, also from Research and Engineering, for supplying most of the data used to compile this report. The remaining data were obtained through the efforts of E. J. Jeanjaquet (Purchasing) and R. A. Minor (Product Definition). Support was provided in the weldability assessment by R. J. Merlini (Component Development Group) and in the formability assessment by S. H. Gelles (S. H. Gelles Associates).
\end{abstract}


RFP-2664 


\title{
SUMMARY OF BERYLLIUM SPECIFICATIONS
}

\author{
Richard R. Corle
}

\begin{abstract}
This report summarizes and compares the main beryllium properties included in producer, Department of Energy (formerly ERDA) facility, and government specifications. These data are tabulated in a sequence established primarily by increasing purity and secondarily by increasing tensile properties, Comments on formability and weldability are also included.
\end{abstract}

\section{INTRODUCTION}

A multitude of beryllium specifications and grade designations has been published to control the chemical, metallurgical, mechanical, and defect limit properties. Many of these specifications (grades) describe the same or equivalent raw materials, but they have been originated and controlled by different authorities. This preponderance of specifications and grades has been a source of confusion for engineering, procurement, and production personnel associated with beryllium products. To alleviate the problem, this report summarizes beryllium specification and grade data.

Comments on formability and weldability have also been included (see Table 1); however, these rating assessments were qualitatively determined and should not be considered irrefutable. The authority for each specification has been identified and is abbreviated as follows:

KBI -Kawecki Berylco Industries, Inc.

Brush - Brush Wellman, Inc.

RF - Rocky Flats Plant, Rockwell International

LLL - Lawrence Livermore Laboratory

DOD - Department of Defense

SAE - Society of Automotive Engineers

For additional information, a few of the more common beryllium powders commercially available have been tabulated in Table 2 of this report. 
TABLE 1. Summary of Beryllium Specifications

BER YLLIUM (\%)

SPEC/GRADE

Revision Year

Authority

Type of Material

$\mathrm{Mfg} / \mathrm{Fab}$

Chem. Comp. (\%)

$\mathrm{Be}(\min )$
$\mathrm{BeO}(\max )$

Beormax

Al (max)
C (max)
Fe (max)
Mg (max)
Si (max)
As Specified (max)
As Specified (max)
As Specified (max)
Other (each, max)
Fe/Al Ratio
Mech. Prop.
F tu (ksi)
F ty (ksi)
Elong. (min \%)
Elev. Temp.

\section{Other}

Avg Grain Size

Max Grain Size

Density $\left(\mathrm{g} / \mathrm{cm}^{3}\right)$

Defect Limits

Weldable ${ }^{\text {,e }}$

Formability

Remarks
$<97$

LS-62

(1976)

\section{KBI}

Sheet

Rolled

$(60$ min-64 max)
1.0 (including
$\left.\mathrm{Al}_{2} \mathrm{O}_{3}\right)$
$\mathrm{Balance}$
0.1
0.1
0.05
--
--
-
0.04

45

30

7
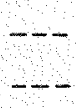

$--$

$---$

99\% Theoretical

Surface Defects Controlled, Note $1^{\mathrm{c}}$

Yes

Good

Trade Name Lockalloy
$<97$

LX-62

(1970)

$\mathrm{KBI}$

Extrusion

Extrude

$(60$ min- 64 max)
1.0 (including
$\mathrm{Al}_{2} \mathrm{O}_{3}$ )
Balance
0.1
0.1
0.05
--
--
-
-
0.04

50

35

7

\section{$--\cdots$}

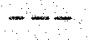

$--$

$---$

2.07

Not Controlled, Note $2^{\mathrm{C}}$

Yes

Good

Trade Name

Lockalloy
$<97$

$\mathrm{HP}-40$

(1975)

KBI

Rod, Bar, Blanks Instrument Grade

Vacuum Hot Press

94

$(4.25 \mathrm{~min})$

0.16

0.25

0.25

0.08

0.15

$--$

$--$

$--$

0.1

$--$

50

$--$

$--$

See Remarks

$\leqslant 10$ microns

$---$

99\% Theoretical

Controlled, Note $3^{c}$

No

No

Microyield

$8 \mathrm{ksimin}$

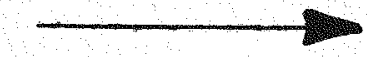

$<97$

$<97$

HP 41

(1975)

KBI

Rod, Bat, Blank Instrument Grade

Vacuum Hot Press

$\mathrm{HPB}^{\mathrm{a}}$

94

(4.25 min)

94

$4.25 \mathrm{~min}-8.0 \mathrm{max}$

$0.16 \quad 0.16$

$0.25 \quad 0.25$

$0.25 \quad 0.3$

$0.08 \quad 0.08$

$0.08 \quad 0.15$

$---\quad--$

$---$

0.1

$-\ldots$

0.1

$--$

$(\mathrm{T})^{\mathrm{b}}(\mathrm{L})^{\mathrm{b}}$

$60 \quad 60$

$45 \quad 45$

$1.5 \quad 1$

$-4$

See Remarks

$\leqslant 12$ microns

$\leqslant 10$ microns

$--$

99\% Theoretical

99\% Theoretical

Controlled, Note $4^{\mathrm{c}}$

Controlled, Note $3^{\mathrm{c}}$

No

No

No

No

Microyield

Reg-8 ksi min Select-9 ksi min
Microyield

$8 \mathrm{ksimin}$

a. HPB - Hot Pressed Block

b. T-Transverse; L-Longitudinal

c. All notes are located at the end of Table 1 .

d. The assessment is made for fusion welding only.

e. The rating assessment for each grade was qualitatively determined and should not be considered irrefutable. 
TABLE 1. cont.

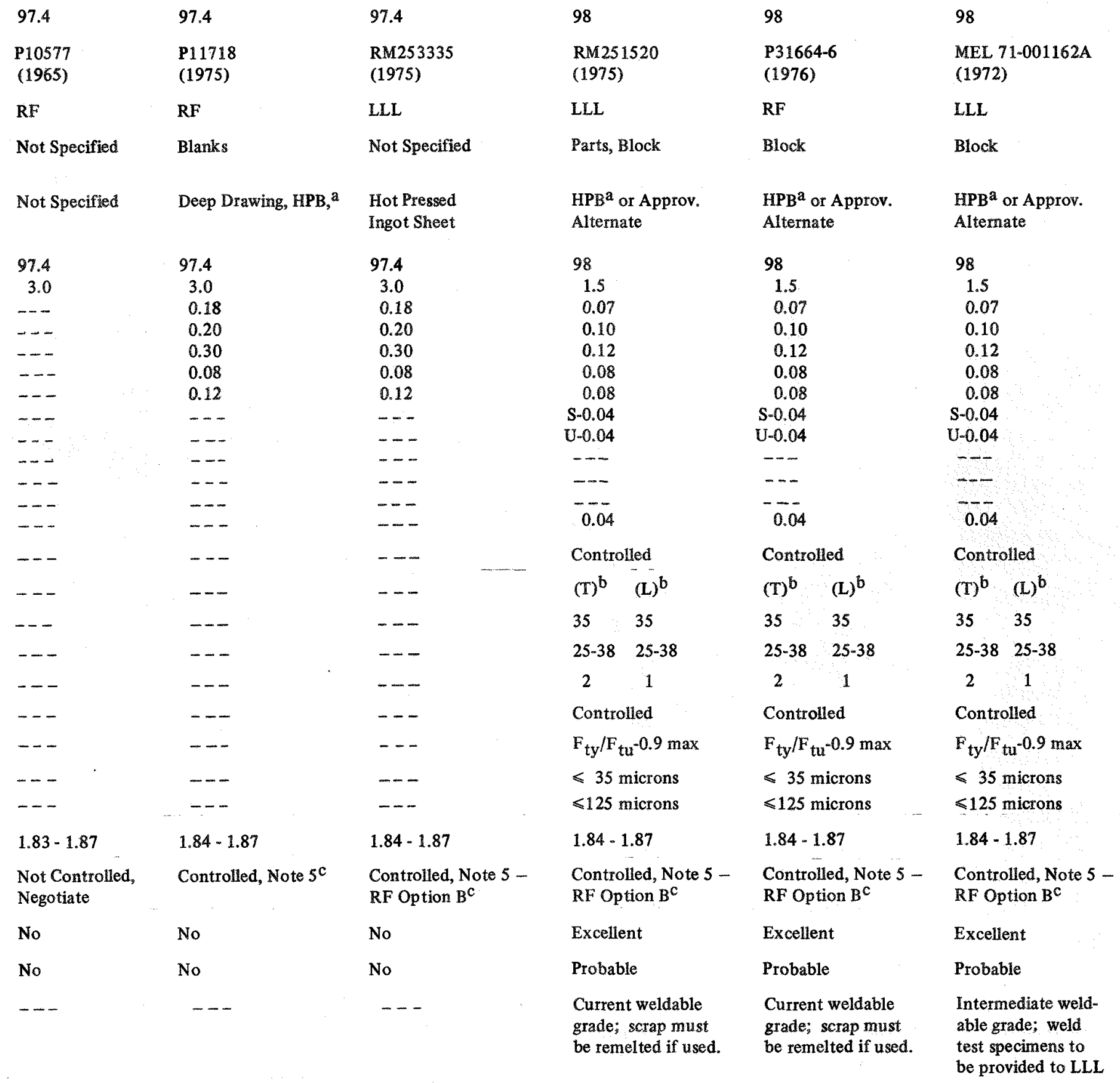

\footnotetext{
a. HPB - Hot Pressed Block

b. T - Transverse; L - Longitudinal

c. All notes are located at the end of Table 1 .
} 
RFP-2664

TABLE 1. cont.

\begin{tabular}{|c|c|c|}
\hline BERYLLIUM $(\%)$ & 98 & 98 \\
\hline $\begin{array}{l}\text { SPEC/GRADE } \\
\text { Revision Year }\end{array}$ & $\begin{array}{l}\text { AMS 7901 } \\
(1961)\end{array}$ & $\begin{array}{l}\text { MIL-B-21531A } \\
\text { (1969) }\end{array}$ \\
\hline Authority & SAE & DOD \\
\hline Type of Material & $\begin{array}{l}\text { Shapes, } \\
\text { Bar, Rod }\end{array}$ & Shapes \\
\hline $\mathrm{Mfg} / \mathrm{Fab}$. & Hot Pressed & Vac. Hot Pressed \\
\hline \multicolumn{3}{|l|}{ Chem. Comp. $(\%)$} \\
\hline $\mathrm{Be}(\mathrm{min})$ & 98 & 98 \\
\hline $\mathrm{BeO}(\max )$ & 2.0 & 2.0 \\
\hline $\mathrm{Al}(\max )$ & 0.20 & 0.16 \\
\hline$C(\max )$ & 0.15 & 0.15 \\
\hline $\mathrm{Fe}(\max )$ & 0.20 & 0.18 \\
\hline $\operatorname{Mg}(\max )$ & 0.08 & 0.08 \\
\hline Si $(\max )$ & 0.12 & 0.08 \\
\hline As Specified (max) & --- & -- \\
\hline As Specified (max) & -- & -- \\
\hline As Specified (max) & --- & $-\infty$ \\
\hline As Specified (max) & -- & -- \\
\hline As Specified (max) & -- & -- \\
\hline Other (each, max) & 0.04 & 0.04 \\
\hline $\mathrm{Fe} / \mathrm{Al}$ Ratio & -- & $-\infty-$ \\
\hline \multicolumn{3}{|l|}{ Mech. Prop. } \\
\hline $\mathrm{F}_{\mathrm{tu}}(\mathrm{ksi})$ & 40 & 40 \\
\hline$F_{\text {ty }}(\mathrm{ksi})$ & 30 & 30 \\
\hline Elong ( $\mathrm{min} \%)$ & 1 & 1 \\
\hline Elev. Temp. & --- & -- \\
\hline other & - & Modulus \\
\hline Avg Grain Size & --- & -- \\
\hline Max Grain Size & --- & --- \\
\hline Density $\left(\mathrm{g} / \mathrm{cm}^{3}\right)$ & $1.84 \mathrm{~min}$ & $1.84 \mathrm{~min}$ \\
\hline Defect Limits & $\begin{array}{l}\text { Workmanship } \\
\text { Clause }\end{array}$ & Controlled, Note 4 \\
\hline Weldable ${ }^{c, d}$ & Limited & Limited \\
\hline Formability $\mathrm{d}^{\mathrm{d}}$ & No & No \\
\hline Remarks & $\begin{array}{l}\text { Powder size } \\
-200 \text { mesh }\end{array}$ & --- \\
\hline
\end{tabular}

98

P11512-E

(1967)

RF

Block, Shape

HPB, ${ }^{a}$ Forged

98

2.0

0.16

0.15

0.20

0.08

0.12

$\mathrm{N}$ (as nitride) 0.06

Mn 0.05

$\mathrm{Cr}, \mathrm{Cu}$
$\mathrm{Ni}, \mathrm{Ti}$$\quad 0.20$ tot.

$--\infty$

0.05

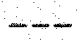

40

30

1
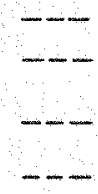

$1.84 \cdot 1.87$

Controlled, Note $5^{b}$

Limited

Poor

Type III $=$ welded based on LLL controls (early weldable grade)
98

P11736-4

(1965)

98

HP-20

(1975)

RF

$\mathrm{KBI}$

Block, machined shapes

Rod, Bar, Blanks

HPB, ${ }^{\text {a Forged }}$

Vac. Hot Pressed

98

2.0

98

0.16

2.0

0.15

0.15

0.15

0.20

0.18

0.08

$\begin{array}{ll}0.12 & 0.08\end{array}$

N (as nitride) 0.06

0.08

Mn 0.05

$\left.\begin{array}{l}\mathrm{Cr}, \mathrm{Cu} \\ \mathrm{Ni}, \mathrm{Ti}\end{array}\right\} 0.20$ tot.

$--$

0.05

$--$

$-+$

$--$

$--$

0.04

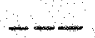

40

30

1

$--1$
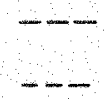

$\leqslant 25$ microns

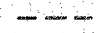

$1.84-1.86$

$99 \%$ Theoretical

Controlled, Note $8^{b}$

Controlled, Note $3^{b}$

Limited

Limited

Poor

Poor

Type WLA $=$ welded based on LASL controls (early weldable grade)

a. HPB - Hot Pressed Block

b. All notes are located at the end of Table 1 .

c. The assessment is made for fusion welding only.

d. The rating assessment for each grade was qualitatively determined and should not be considered irrefutable. 
TABLE 1. cont.

\begin{tabular}{|c|c|c|c|c|c|c|c|c|c|}
\hline 98 & \multicolumn{2}{|l|}{98} & \multicolumn{2}{|l|}{98} & \multicolumn{2}{|l|}{98} & \multicolumn{2}{|l|}{98} & 98 \\
\hline $\begin{array}{l}\text { S-200-E } \\
(1972)\end{array}$ & \multicolumn{2}{|c|}{$\begin{array}{l}\text { SS229054 } \\
(1972)\end{array}$} & \multicolumn{2}{|c|}{$\begin{array}{c}\text { P31351 } \\
(1973)\end{array}$} & \multicolumn{2}{|c|}{$\begin{array}{l}\text { S-245 } \\
(1972)\end{array}$} & \multicolumn{2}{|c|}{$\begin{array}{l}\text { HP-21 } \\
(1975)\end{array}$} & $\begin{array}{l}\text { HP-22 } \\
(1975)\end{array}$ \\
\hline Brush & \multicolumn{2}{|l|}{ LLL } & \multicolumn{2}{|l|}{$\mathrm{RF}$} & \multicolumn{2}{|c|}{ Brush } & \multicolumn{2}{|l|}{$\mathrm{KBI}$} & KBI \\
\hline Parts, Shapes & \multicolumn{2}{|l|}{ Parts } & \multicolumn{2}{|l|}{ Parts } & \multicolumn{2}{|c|}{ Block } & \multicolumn{2}{|c|}{$\begin{array}{l}\text { Rod, Bar, Billet, } \\
\text { Blank, Structural } \\
\text { Grade }\end{array}$} & $\begin{array}{l}\text { Rod, Bar, Billet, } \\
\text { Blank, Instrument } \\
\text { Grade }\end{array}$ \\
\hline Hot Pressed & \multicolumn{2}{|c|}{$\begin{array}{l}\text { HPB, }{ }^{a} \text { Powder, } \\
\text { Forgings, CRS, } \\
\text { Extrusion }\end{array}$} & \multicolumn{2}{|c|}{$\begin{array}{l}\text { HPB }^{\mathrm{a}} \text {, Powder, } \\
\text { Forgings, CRS, } \\
\text { Extrusion }\end{array}$} & \multicolumn{2}{|c|}{$\mathrm{HPB}^{\mathrm{a}}$} & \multicolumn{2}{|c|}{ Vacuum Hot Pressed } & Vacuum Hot Pressed \\
\hline 98 & \multicolumn{2}{|l|}{98} & \multicolumn{2}{|l|}{98} & \multicolumn{2}{|l|}{98} & \multicolumn{2}{|l|}{98} & 98 \\
\hline 2.0 & \multicolumn{2}{|l|}{2.0} & \multicolumn{2}{|l|}{2.0} & \multicolumn{2}{|l|}{2.0} & \multicolumn{2}{|c|}{2.0} & 2.0 \\
\hline 0.16 & 0.16 & & 0.16 & & 0.10 & & 0.15 & & 0.15 \\
\hline 0.15 & 0.15 & & 0.15 & & 0.15 & & 0.1 & & 0.15 \\
\hline 0.18 & 0.20 & & 0.20 & & 0.15 & & 0.18 & & 0.20 \\
\hline 0.08 & 0.08 & & 0.08 & & 0.08 & & 0.0 & & 0.08 \\
\hline 0.08 & 0.08 & & 0.08 & & 0.08 & & 0.08 & & 0.08 \\
\hline--- & -- & & -- & & -- & & -- & & --- \\
\hline--- & -- & & -- & & -- & & -- & & --- \\
\hline-- & -- & & -- & & -- & & - & & --- \\
\hline--- & -- & & $-\cdots$ & & -- & & $-\cdots$ & & $-\cdots$ \\
\hline-- & -- & & $--\cdots$ & & -- & & -- & & $--\infty$ \\
\hline 0.04 & 0.04 & & 0.04 & & 0.04 & & 0.0 & & 0.04 \\
\hline--- & $-\cdots$ & - & --- & & -- & & -- & & -- \\
\hline-- & $(\mathrm{T})^{\mathrm{c}}$ & $(L)^{c}$ & $(T)^{c}$ & $(L)^{c}$ & $(\mathrm{~T})^{\mathrm{c}}$ & $(L)^{c}$ & $(T)^{c}$ & $(L)^{c}$ & $--\infty$ \\
\hline 40 & 45 & 40 & 45 & 40 & 45 & 45 & 45 & 40 & 45 \\
\hline 30 & 35 & 30 & 35 & 30 & 35 & 30 & 35 & 35 & 35 \\
\hline 1 & 2 & 1.5 & 2 & 1.5 & 2 & 1.5 & 2 & 1.5 & 2 \\
\hline$-\cdots--$ & -- & & --1 & & $-\cdots$ & & -- & & --- \\
\hline-- & -- & & $-\cdots$ & & --- & & - & & See Remarks \\
\hline$\leqslant 25$ microns & $-\cdots$ & & -- & & $\leqslant 25$ & nicrons & $\leqslant 25$ & hicrons & $\leqslant 25$ microns \\
\hline$\cdots-$ & -- & & -- & & -- & & -- & & --- \\
\hline $1.84 \mathrm{~min}$ & & & 184 & & 1.84 & & $99 \%$ & leoretical & 99\% Theoretical \\
\hline Controlled, Note $3^{\mathrm{d}}$ & & & Contr & led & Cont 1 & lled, Note $3^{\mathrm{d}}$ & Cont & lled, Note $3^{\mathrm{d}}$ & Controlled, Note $3^{\mathrm{d}}$ \\
\hline Limited & Limit & & Limit & & Limit & & Limi & & Limited \\
\hline Poor & Poor & & Poor & & Poor & & Poor & & Poor \\
\hline--- & $\begin{array}{l}\text { Spec } \\
\text { and } 1 \\
\text { cont }\end{array}$ & $\begin{array}{l}\text { ed part } \\
\text { cess } \\
\text { l imposed }\end{array}$ & $\begin{array}{l}\text { Speci } \\
\text { and } p \\
\text { contr }\end{array}$ & $\begin{array}{l}\text { ed part } \\
\text { impess } \\
\text { imposed }\end{array}$ & -- & & -- & & Microyield $5 \mathrm{ksi} \min$ \\
\hline
\end{tabular}

a. HPB - Hot Pressed Block

b. CRS - Cross Rolled Sheet

c. T-Transverse; L - Longitudinal

d. All notes are located at the end of Table 1. 
TABLE 1. cont.

\begin{tabular}{|c|c|c|c|c|c|}
\hline BERYLLIUM (\%) & 98 & 98 & 98 & 98 & 98 \\
\hline $\begin{array}{l}\text { SPEC/GRADE } \\
\text { Revision Year }\end{array}$ & $\begin{array}{l}\mathrm{I}-220 \\
(1974)\end{array}$ & $\begin{array}{l}\text { PR-200-E } \\
\text { (1972) }\end{array}$ & $\begin{array}{l}\text { PR-20 } \\
\text { (1968) }\end{array}$ & $\begin{array}{l}\text { XT-20 } \\
(1974)\end{array}$ & $\begin{array}{l}\text { SR-200 } \\
\text { (1974) }\end{array}$ \\
\hline Authority & Brush & Brush & KBI & KBI & Brush \\
\hline Type of Material & Block, Inst. Grade & Plate & Plate & Extrusion & Sheet \\
\hline Mfg./Fab. & $\mathrm{HPB}^{\mathrm{a}}$ & Hot Roll, HPB ${ }^{a}$ & Cross Rolling Block & Extrude & Hot Ro \\
\hline \multicolumn{6}{|l|}{ Chem. Comp. (\%) } \\
\hline $\mathrm{Be}(\min )$ & 98 & 98 & 98 & 98 & 98 \\
\hline $\mathrm{BeO}(\max )$ & 2.0 & 2.0 & 2.0 & 2.0 & 2.0 \\
\hline $\mathrm{A} 1(\max )$ & 0.10 & 0.16 & 0.15 & 0.18 & 0.16 \\
\hline$C(\max )$ & 0.15 & 0.15 & 0.15 & 0.15 & 0.15 \\
\hline $\mathrm{Fe}(\max )$ & 0.15 & 0.18 & 0.18 & 0.20 & 0.18 \\
\hline $\mathbf{M g}(\max )$ & 0.08 & 0.08 & 0.08 & 0.08 & 0.08 \\
\hline $\mathrm{Si}(\max )$ & 0.08 & 0.08 & 0.08 & 0.08 & 0.08 \\
\hline As Specified (max) & --- & $\ldots$ & -- & --- & --- \\
\hline As Specified (max) & -- & --- & $-\cdots$ & -- & --- \\
\hline As Specified (max) & --- & -- & --- & -- & -- \\
\hline As Specified (max) & -- & $\ldots$ & -- & -- & --- \\
\hline As Specified (max) & --- & $\ldots$ & -- & --- & -- \\
\hline Other (each, max) & 0.04 & 0.04 & 0.04 & 0.04 & 0.04 \\
\hline $\mathrm{Fe} / \mathrm{Al}$ Ratio & -- & -- & $-\cdots$ & -- & -- \\
\hline Mech. Prop. & -- & (T) $\quad(\mathrm{L})$ & (T) (L) & -- & -- \\
\hline $\mathrm{F}_{\mathrm{tu}}(\mathrm{ksi})$ & 45 & $65^{\mathrm{b}} \quad 60^{\mathrm{c}}$ & $65^{\mathrm{b}} \quad 60^{\mathrm{c}}$ & 70 & 70 \\
\hline$F_{\text {ty }}(\mathrm{ksi})$ & 35 & $45: 40$ & $45 \quad 40$ & 45 & 50 \\
\hline Elong. $(\min \%)$ & 2 & $4 \quad 3$ & $4 \quad 3$ & 5 & 10 \\
\hline Elev. Temp. & -- & --- & --- & -- & -- \\
\hline Other & See Remarks & -- & --- & --- & --- \\
\hline Avg Grain Size & $\leqslant 25$ microns & --- & --- & -- & -- \\
\hline Max Grain Size & --- & $-\cdots$ & --- & $-\cdots$ & -- \\
\hline Density $\left(\mathrm{g} / \mathrm{cm}^{3}\right)$ & $1.84 \mathrm{~min}$ & --- & $1.84 \mathrm{~min}$ & $1.84 \mathrm{~min}$ & -- \\
\hline Defect Limits & Controlled, Note $3^{\mathrm{d}}$ & $\begin{array}{l}\text { Controlled If Speci- } \\
\text { fied, Note } 4^{d}\end{array}$ & $\begin{array}{l}\text { Penetrant Inspection } \\
\text { If Required }\end{array}$ & Controlled, Note $4^{\mathrm{d}}$ & -- \\
\hline Weldable, $\mathrm{f}$ & Limited & Limited & Limited & -- & Limited \\
\hline Formability $\mathrm{f}$ & Poor & Poor & Poor & No & Poor \\
\hline Remarks & Microyield $5 \mathrm{ksi}$ min & $-\ldots$ & -- & $-\cdots$ & $\ldots$ \\
\hline
\end{tabular}

a. HPB - Hot Pressed Block

b. Thickness of 0.250 to 0.450 inch

c. Thickness of 0.451 to 0.600 inch

d. All notes are located at the end of Table 1 .

e. The assessment is made for fusion welding only.

f. The rating assessment for each grade was qualitatively determined and should not be considered irrefutable. 
TABLE 1. cont.

\begin{tabular}{|c|c|c|c|c|c|}
\hline 98 & 98 & 98 & 98.5 & 98.5 & 98.5 \\
\hline PS-20 & PF-20 & $\begin{array}{l}\text { HIP-50 } \\
\text { (preproduction grade) }\end{array}$ & S-100-E & HP-12 & HP-10 \\
\hline (1975) & (1975) & (1977) & (1972) & (1968) & (1971) \\
\hline $\mathrm{KBI}$ & KBI & KBI & Brush & Brush & $\mathrm{KBI}$ \\
\hline Sheet & Foil & Block, Bar, Rod & Parts, Shapes & Blocks & $\begin{array}{l}\text { Block, Thermal } \\
\text { Cycling }\end{array}$ \\
\hline Cross Roll HPB ${ }^{\mathrm{a}}$ & Produced from $\mathrm{HPB}^{\mathrm{a}}$ & $\begin{array}{l}\text { Hot Isostatic Consolida- } \\
\text { tion of Electrolytic } \\
\text { Flake Powders }\end{array}$ & Hot Pressed & Hot Pressed & $\mathrm{HPB}^{\mathrm{a}}$ \\
\hline 98 & 98 & 98 & 98.5 & 98.5 & 98.5 \\
\hline 2.0 & 2.0 & 1.6 & 1.2 & 1.2 & 1.2 \\
\hline 0.15 & 0.09 & 0.0075 & 0.14 & 0.09 & 0.08 \\
\hline 0.15 & 0.12 & 0.003 & 0.15 & 0.12 & 0.10 \\
\hline 0.18 & 0.15 & 0.005 & 0.16 & 0.04 & 0.15 \\
\hline 0.08 & 0.08 & 0.003 & 0.08 & 0.06 & 0.08 \\
\hline 0.08 & 0.08 & 0.0045 & 0.08 & 0.08 & 0.08 \\
\hline-- & --- & Cr 0.0035 & -- & $\ldots$ & $-\ldots$ \\
\hline--- & -- & Cu 0.0070 & --- & -- & -- \\
\hline-- & --- & $\mathrm{Mn} 0.0025$ & --- & -- & $-\infty$ \\
\hline--- & --- & $\mathrm{Ni} 0.001$ & --- & -- & $-\infty$ \\
\hline--- & --- & --- & --- & $-\cdots$ & $-\cdots$ \\
\hline 0.04 & 0.04 & --- & 0.04 & 0.04 & 0.04 \\
\hline$-\cdots$ & -- & --- & --- & -- & --- \\
\hline-- & -- & -- & -- & --- & $-\cdots$ \\
\hline 70 & $-\cdots$ & 80)$. & 35 & 35 & 37 \\
\hline 50 & --- & $60\}$ typical & 27 & 27 & 24 \\
\hline 10 & $-\ldots$ & 4) & 1 & 1 & 1.5 \\
\hline-- & --- & -- & --- & -- & -- \\
\hline--- & --- & -- & -- & -- & -- \\
\hline-- & $-\cdots$ & -- & $\leqslant 25$ microns & $<25$ microns & $\leqslant 25$ microns \\
\hline-- & -- & --- & --- & --- & -- \\
\hline 99\% Theoretical & 99\% Theoretical & --- & $1.84 \mathrm{~min}$ & $1.94 \mathrm{~min}$ & 99\% Theoretical \\
\hline $\begin{array}{l}\text { Controlled, Note } 3 \text { - } \\
\text { Type } 2^{\mathrm{b}}\end{array}$ & $\begin{array}{l}\text { Vacuum Tight, } \\
\text { Optically Dense }\end{array}$ & -- & Controlled, Note $3^{b}$ & Controlled, Note $3^{b}$ & Controlled, Note $4^{b}$ \\
\hline Limited & Limited & Unknown & Good & Good & Good \\
\hline Poor & Moderate & Unknown & Moderate & Moderate & Moderate \\
\hline--- & --- & Microyield 8 ksi & -- & --- & -- \\
\hline
\end{tabular}

a. HPB - Hot Pressed Block

b. All notes are located at the end of Table 1 . 
TABLE 1. cont.

\begin{tabular}{|c|c|c|c|c|c|}
\hline BERYLLIUM (\%) & 98.5 & 98.5 & 99 & 99 & 99 \\
\hline $\begin{array}{l}\text { SPEC/GRADE } \\
\text { Revision Year }\end{array}$ & $\begin{array}{l}\text { S-65 } \\
(1973)\end{array}$ & $\begin{array}{l}\text { MEL 76-001319 } \\
\text { (1976) }\end{array}$ & $\begin{array}{l}\text { C-50 } \\
(1968)\end{array}$ & $\begin{array}{l}\text { IF-2 } \\
(1975)\end{array}$ & $\begin{array}{l}\text { HP-8 } \\
(1968)\end{array}$ \\
\hline Authority & Brush & LLL & $\mathrm{KBI}$ & KBI & KBI \\
\hline Type of Material & $\begin{array}{l}\text { Block, Structural } \\
\text { Grade }\end{array}$ & Parts, Shapes & Ingot & Foil & Block, Shapes \\
\hline Mfg./Fab. & $\mathrm{HPB}^{\mathrm{a}}$ & $\mathrm{HPB}^{\mathrm{a}} \mathrm{HIP}^{\mathrm{b}}$ & $\begin{array}{l}\text { Vacuum Melt } \\
\text { and Cast }\end{array}$ & $\begin{array}{l}\text { Extrude and Roll } \\
\text { Vacuum Cast Ingot }\end{array}$ & Hot Pressed \\
\hline \multicolumn{6}{|l|}{ Chem. Comp. (\%) } \\
\hline $\mathrm{Be} \quad(\min )$ & 98.5 & 98.5 & 99 & 99 & 99 \\
\hline $\mathrm{BeO}(\max )$ & 1.0 & 1.2 & 0.5 & 0.03 & 0.9 \\
\hline Al (max) & 0.06 & 0.06 & 0.09 & 0.09 & 0.07 \\
\hline C $\quad(\max )$ & 0.12 & 0.1 & 0.10 & 0.10 & 0.10 \\
\hline $\mathrm{Fe} \quad(\max )$ & 0.12 & 0.1 & 0.15 & 0.15 & 0.075 \\
\hline $\mathrm{Mg}(\max )$ & 0.08 & 0.08 & 0.08 & 0.02 & 0.06 \\
\hline $\mathrm{Si} \quad(\max$ & 0.06 & 0.06 & 0.08 & 0.08 & 0.06 \\
\hline Cr (max) & -- & --- & 0.01 & --- & 0.01 \\
\hline Co $(\max )$ & $\ldots$ & $\ldots$ & 0.0005 & --- & 0.0005 \\
\hline $\mathrm{Cu} \quad(\max )$ & --- & --- & 0.015 & --- & 0.015 \\
\hline $\mathrm{Pb} \quad(\max )$ & $\ldots$ & --- & 0.005 & $-\ldots$ & 0.002 \\
\hline $\mathrm{Mn}(\max )$ & --- & -- & 0.015 & $-\cdots$ & 0.012 \\
\hline Mo (max) & $\ldots$ & $\ldots$ & 0.002 & - - - & 0.002 \\
\hline $\mathrm{Ni} \quad(\max )$ & --- & $\ldots$ & 0.03 & --- & 0.02 \\
\hline B $\quad(\max )$ & $\ldots$ & $\ldots$ & 0.0002 & $\ldots$ & 0.0002 \\
\hline $\mathrm{Cd}(\max )$ & $\ldots$ & $\ldots$ & 0.0002 & $\ldots$ & 0.0002 \\
\hline $\mathrm{Ca}(\max )$ & $\ldots$ & $\ldots$ & 0.02 & $\ldots$ & 0.01 \\
\hline $\mathrm{Li} \quad(\max )$ & $\ldots$ & $-\ldots$ & 0.0003 & $\ldots$ & 0.0003 \\
\hline $\mathrm{N} \quad(\max )$ & $\ldots$ & - & 0.03 & $\ldots$ & 0.02 \\
\hline $\mathrm{Ag} \quad(\max )$ & $-\cdots$ & $-\ldots$ & 0.001 & $\ldots$ & 0.001 \\
\hline As Specified (max) & $\ldots$ & $S=0.04$ & $\mathrm{Zn} 0.02$ & $\ldots$ & $-\ldots$ \\
\hline As Specified (max) & $\ldots$ & $\mathrm{U}-0.04$ & $\therefore--$ & --- & --- \\
\hline As Specified (max) & --- & --- & -- & --- & $-\cdots$ \\
\hline As Specified (max) & $-\ldots$ & $\ldots$ & --- & -- & --- \\
\hline Other (each, max) & 0.04 & 0.04 & --- & 0.04 & --- \\
\hline $\mathrm{Fe} /$ Al Ratio & $\ldots$ & Controlled & --- & $-\cdots$ & -- \\
\hline Mech. Prop. & $\ldots$ & $(\mathrm{T} \& \mathrm{~L})^{\mathrm{c}}$ & --- & --- & $\ldots-$ \\
\hline $\mathrm{F}_{\mathrm{tu}}(\mathrm{ksi})$ & 42 & 50 & -- & -- & 35 \\
\hline $\mathrm{F}_{\text {ty }}(\mathrm{ksi})$ & 30 & $35-50$ & -- & -- & 25 \\
\hline Elong $(\min \%)$ & 3 & 3 & --- & --- & 1 \\
\hline Elev. Temp. & & Controlled & & & \\
\hline Other & --- & -- & -- & -- & --- \\
\hline Avg Grain Size & $\leqslant 15$ microns & $\leqslant 15$ microns & -- & --- & $-\cdots$ \\
\hline Max Grain Size & --- & $\ldots$ & --1 & --- & -- \\
\hline Density $\left(\mathrm{g} / \mathrm{cm}^{3}\right)$ & 99\% Theoretical & 99\% Theoretical & $-\cdots$ & 1.83 & $1.84 \mathrm{~min}$ \\
\hline Defect Limits & $\begin{array}{l}\text { Controlled } \\
\text { Note } 3 \text { - Type } 1^{\text {d }}\end{array}$ & Controlled, Note $6^{d}$ & $-\ldots$ & $\begin{array}{l}\text { Vacuum Tight, } \\
\text { Optically Dense }\end{array}$ & Controlled, Note $4^{\mathrm{d}}$ \\
\hline Weldable e,f & Good & Excellent & Good & Good & Good \\
\hline Formability $f$ & Moderate & Moderate & Good & Moderate & Moderate \\
\hline Remarks & -- & -- & --- & --- & $-\cdots$ \\
\hline \multicolumn{3}{|c|}{$\begin{array}{l}\text { b. HIP - Hot Isostatically Pressed } \\
\text { c. T-Transverse; } L \text { - Longitudinal } \\
\text { d. All notes are located at the end of Table } 1 . \\
\text { e. The assessment is made for fusion welding only. } \\
\text { f. The rating assessment for each grade was qualitatively determined } \\
\text { and should not be considered irrefutable. }\end{array}$} & . & & \\
\hline
\end{tabular}


TABLE 1. cont.

\begin{tabular}{|c|c|c|c|c|c|}
\hline 99 & 99 & 99 & 99 & 99 & 99 \\
\hline $\begin{array}{l}\text { N-50-C } \\
(1972)\end{array}$ & $\begin{array}{l}\text { HP-81 } \\
(1975)\end{array}$ & $\begin{array}{l}1-70 \mathrm{~A} \\
(1973)\end{array}$ & $\begin{array}{l}8115000 \\
(1973)\end{array}$ & $\begin{array}{l}\text { CIP-HIP-1 } \\
\text { (1975) }\end{array}$ & $\begin{array}{l}\text { CD-11 } \\
(1966)\end{array}$ \\
\hline Brush & KBI & Brush & $\mathrm{RF}$ & $\mathrm{KBI}$ & KBI \\
\hline Block, Billet, Shapes & Block, Optical Grade & Block, Optical Grade & Sheet & $\begin{array}{l}\text { Block, Rod, } \\
\text { Bar, Blanks }\end{array}$ & Wire \\
\hline Not Specified & Vacuum Hot Pressed & Hot Pressed & $\begin{array}{l}\text { Vacuum Melt, Cast } \\
\text { and Rolled Ingot }\end{array}$ & $\begin{array}{l}\text { Isostatic } \\
\text { Consolidated }\end{array}$ & Warm Draw Rod \\
\hline 99 & 99 & 99 & 99 & 99 & 99 \\
\hline 0.9 & 1.0 & 0.7 & 0.05 & 1.2 & 0.02 \\
\hline 0.06 & 0.08 & 0.07 & 0.12 & 0.01 & 0.09 \\
\hline 0.10 & 0.10 & 0.07 & 0.12 & 0.0300 & 0.1 \\
\hline 0.075 & 0.12 & 0.10 & 0.25 & 0.0300 & 0.15 \\
\hline 0.05 & 0.08 & 0.07 & 0.05 & 0.0060 & 0.08 \\
\hline 0.06 & 0.08 & 0.07 & 0.12 & 0.0100 & 0.08 \\
\hline 0.01 & --- & --- & 0.05 & 0.0025 & --- \\
\hline 0.0005 & -- & $\ldots$ & --- & 0.0005 & --- \\
\hline 0.015 & --- & -- & 0.05 & 0.0050 & $\ldots$ \\
\hline 0.002 & $\ldots$ & --- & $-\ldots$ & 0.0005 & $\ldots$ \\
\hline 0.012 & $\ldots$ & $-\ldots$ & -- & 0.0030 & --- \\
\hline 0.002 & --- & $-\cdots$ & $\ldots$ & 0.0010 & $\ldots$ \\
\hline 0.02 & --- & --- & 0.05 & 0.0200 & --- \\
\hline 0.0002 & --- & --- & --- & --- & --- \\
\hline 0.0002 & $-\cdots$ & $--\infty$ & $-\cdots$ & -- & $-\cdots$ \\
\hline 0.01 & -- & -- & -- & --- & --- \\
\hline 0.0003 & -- & $-\cdots$ & --- & $\ldots$ & --- \\
\hline 0.02 & --- & -- & 0.06 & --- & -- \\
\hline 0.0010 & $--\overline{0}$ & --- & --- & --- & $=-$ \\
\hline$\overline{-z}$ & $\begin{array}{l}0.04 \\
---\end{array}$ & 0.04 & 0.02 & $=z$ & $=-$ \\
\hline--- & -- & -- & --- & --- & -- \\
\hline$\overline{z--}$ & -- & $\overline{---}$ & Ti 0.05 & -- & -- \\
\hline & & & -- & -- & --- \\
\hline--- & --- & -- & Controlled & --- & --- \\
\hline-- & $\ldots$ & --- & $(T)^{\mathrm{a}}(\mathrm{L})^{\mathrm{a}}$ & --- & $\leqslant 0.010$-in. dia. \\
\hline 35 & 35 & 35 & $38 \quad 40$ & 50 & 130 \\
\hline 25 & 25 & 25 & 22 & 35 & 110 \\
\hline 1 & 2 & 2 & 33 & 3 & 1 \\
\hline-- & See Remarks & See Remarks & $F_{t u} L \& T$ within $10 \mathrm{ksi}$ & --- & --- \\
\hline--- & $\leqslant 30$ microns & $\leqslant 20$ microns & --- & $\leqslant 15$ microns & --- \\
\hline-- & --- & -- & --- & --- & $-\cdots$ \\
\hline $1.84 \mathrm{~min}$ & 99\% Theoretical & 99\% Theoretical & $1.84-1.86$ & $99 \%$ Theoretical & 1.84 \\
\hline Controlled, Note $4^{\mathrm{b}}$ & $\begin{array}{l}\text { Controlled, } \\
\text { Note } 3 \text { - Type } 2^{\text {b }}\end{array}$ & $\begin{array}{l}\text { Controlled, } \\
\text { Note } 3 \text {-Type } 1^{b}\end{array}$ & Controlled, Note $7^{\mathrm{b}}$ & $\begin{array}{l}\text { Controlled, } \\
\text { Note } 3 \text { - Type } 2^{b}\end{array}$ & --- \\
\hline Good & Good & Good & Excellent & Limited & --- \\
\hline \multirow[t]{2}{*}{ Moderate } & Moderate & Moderate & Good & Moderate & Moderate \\
\hline & Microyield Reported & $\begin{array}{l}\text { Microyield Thermal } \\
\text { Expansion Reported }\end{array}$ & & $\begin{array}{l}\text { Origin of Powder- } \\
\text { Electrolytic Flake }\end{array}$ & $\begin{array}{l}\text { Strength for } \\
\text { Larger Diameter } \\
\text { Wire Available }\end{array}$ \\
\hline
\end{tabular}

a. T - Transverse; L - Longitudinal

b. All notes are located at the end of Table 1 . 
TABLE 1. cont.

\begin{tabular}{|c|c|c|c|c|}
\hline BERYLLIUM $(\%)$ & 99.5 & 99.8 & 99.8 & 99.8 \\
\hline $\begin{array}{l}\text { SPEC/GRADE } \\
\text { Revision Year }\end{array}$ & $\begin{array}{l}\text { XT-1 } \\
\text { (1975) }\end{array}$ & $\begin{array}{l}C-1 \\
(1976)\end{array}$ & $\begin{array}{l}\text { 1S-1 } \\
(1975)\end{array}$ & $\begin{array}{l}1 \mathrm{~F}-1 \\
(1975)\end{array}$ \\
\hline Authority & KBI & KBI & KBI & KBI \\
\hline Type of Material & Extrusion & Ingot & Sheet & Foil \\
\hline $\mathrm{Mfg} / \mathrm{Fab}$. & $\begin{array}{l}\text { Extruded Vacuum Melt } \\
\text { and Cast Ingot }\end{array}$ & Vacuum Melt and Cast & $\begin{array}{l}\text { Extruded and Cross } \\
\text { Roll Ingots }\end{array}$ & $\begin{array}{l}\text { Extruded and Hot } \\
\text { Roll Ingots }\end{array}$ \\
\hline \multicolumn{5}{|l|}{ Chem. Comp. (\%) } \\
\hline Be $(\min )$ & 99.5 & 99.8 & 99.8 & 99.8 \\
\hline $\mathrm{BeO}(\max )$ & 0.03 & 0.03 & 0.03 & 0.03 \\
\hline Al (max) & 0.01 & 0.01 & 0.01 & 0.01 \\
\hline C $(\max )$ & 0.03 & 0.03 & 0.03 & 0.03 \\
\hline $\mathrm{Fe} \quad(\max )$ & 0.03 & 0.03 & 0.03 & 0.03 \\
\hline $\mathrm{Mg}(\max )$ & 0.006 & 0.006 & 0.006 & 0.006 \\
\hline $\mathrm{Si}(\max )$ & 0.010 & 0.01 & 0.01 & 0.01 \\
\hline $\mathrm{Cr} \quad(\max )$ & 0.0025 & 0.0025 & 0.0025 & 0.0025 \\
\hline Co $(\max )$ & 0.0005 & 0.0005 & 0.0005 & 0.0005 \\
\hline $\mathrm{Cu} \quad(\max )$ & 0.005 & 0.005 & 0.005 & 0.005 \\
\hline $\mathrm{Pb} \quad(\max )$ & 0.0005 & 0.0005 & 0.0005 & 0.0005 \\
\hline Mn $(\max )$ & 0.003 & 0.003 & 0.003 & 0.003 \\
\hline Mo $(\max )$ & 0.001 & 0.001 & 0.001 & 0.001 \\
\hline $\mathrm{Ni} \quad(\max )$ & 0.02 & 0.02 & 0.02 & 0.02 \\
\hline B $(\max )$ & --- & -- & --- & --- \\
\hline $\mathrm{Cd}(\max )$ & -- & --- & --- & --- \\
\hline $\mathrm{Ca}$ (max) & $-\ldots$ & 0.02 & 0.02 & 0.02 \\
\hline $\mathrm{Li} \quad(\max )$ & --- & $\ldots$ & --- & --- \\
\hline $\mathrm{N} \quad(\max )$ & --- & --- & --- & --- \\
\hline $\mathrm{Ag} \quad(\max )$ & $-\cdots$ & 0.0005 & 0.0005 & 0.0005 \\
\hline As Specified (max) & $-\ldots$ & $\mathrm{Zn} 0.01$ & $\mathrm{Zn} 0.01$ & $\mathrm{Zn} 0.01$ \\
\hline As Specified (max) & $-\infty$ & Ti 0.001 & Ti 0.001 & Ti 0.001 \\
\hline Other (each, max) & --- & --- & -- & $\ldots$ \\
\hline Fe/Al Ratio & $-\infty$ & --- & $-\cdots$ & $-\cdots$ \\
\hline Mech. Prop. & -- & -- & --- & --- \\
\hline$F_{\text {tu }}(\mathrm{ksi})$ & Reported & -- & (Depends on gage) & --- \\
\hline$F_{t y}(k s i)$ & Reported & -- & $\ldots$ & -- \\
\hline Elong. (min \%) & Reported & $\ldots$ & $\begin{array}{l}\text { Not Specified, } \\
\text { Controlled }\end{array}$ & -- \\
\hline Avg Grain Size & --- & -- & -- & -- \\
\hline Max Grain Size & -- & -- & --- & --- \\
\hline Density $\left(\mathrm{g} / \mathrm{cm}^{3}\right)$ & $1.83 \mathrm{~min}$ & $\ldots-$ & $1.83 \mathrm{~min}$ & $1.83 \mathrm{~min}$ \\
\hline Defects & -- & -- & $\begin{array}{l}\text { Controlled, } \\
\text { Note } 3 \text { - Type } 2^{\mathrm{a}}\end{array}$ & $\begin{array}{l}\text { Vacuum Tight, } \\
\text { Optically Dense }\end{array}$ \\
\hline Weldable & Good & --- & Good & Good \\
\hline Formability $\mathrm{c}$ & Good & --- & Good & Good \\
\hline Remarks & --- & $\ldots$ & --- & $\ldots$ \\
\hline
\end{tabular}

a. All notes are located at the end of Table 1.

b. The assessment is made for fusion welding only.

c. The rating assessment for each grade was qualitatively determined and should not be considered irrefutable. 
TABLE 1. cont.

\section{NOTES*}

\section{Penetrant Inspection}

The surface of the sheet in the finished condition prior to shipment shall be free from cracks and other defects as determined by liquid penetrant inspection.

2. Penetrant and radiographic inspection will be performed when required.

\section{Penetrant Inspection}

The surface of the material in the machined or machined and etched condition shall be free of porosity or cracks as determined by liquid penetrant inspection.

\section{Radiographic Inspection}

The material shall be radiographically inspected for conformance to Type 1 or Type 2 Qualify with regard to radiographic indications (voids and/or inclusions).

Type 1 and Type 2 Qualify are defined as follows:

Maximum Dimension of Radiographic Indications

The largest dimension of an indication mea sured in the plane of the radiograph shall not exceed 0.030 inch ( 0.76 millimeter).

Maximum Average Dimension of Radiographic Indications

The arithmetic average of the maximum and the minimum dimensions of an indication

*These criteria are not necessarily exactly as stated in each individual specification; however, they are comparative. measured in the plane of a radiograph shall not exceed 0.020 inch ( 0.51 millimeter).

Total Combined Value of Radiographic Indications

In any one cubic inch $\left(1.65 \times 10^{4}\right.$ cubic millimeter) of material, the total combined volume of all indications larger than 0.001 inch ( 0.025 millimeter) shall not exceed the volume of spheres having the following diameters:

\section{Diameter of Sphere}

Volume

Quality [in. (mm)] [cu in. $\left.\times 10^{-6}\left(\mathrm{~mm}^{3}\right)\right]$

$\begin{array}{lll}\text { Type } 1 & 0.050(1.27) & 66(1.07) \\ \text { Type } 2 & 0.032(0.81) & 17(0.28)\end{array}$

The volume of the indications shall be calculated on the basis of an assumed sphere, the diameter of which is equal to the maximum average dimension of the indication.

4. Penetrant Inspection

Liquid penetrant inspection of machined surfaces shall reveal no porosity or cracks.

Radiographic Inspection

The combined volume per cubic inch of voids and inclusions shall not exceed the volume of a sphere 0.050 inches in diameter.

\section{Surface Defects}

When examined visually, shapes shall be entirely free of detectable surface defects such as cracks, porosity, and scale. 
TABLE 1. cont.

Radiographic Inspection

\section{RF Option A -}

High density inclusions and/or internal or exposed cavities in each part shall be limited to the following:

\begin{tabular}{|c|c|c|}
\hline $\begin{array}{c}\text { No. Cavities } \\
\text { or Inclusions } \\
\text { Allowed } \\
\end{array}$ & $\begin{array}{c}\text { Nominal } \\
\text { Dimension } \\
\text { (in.) }\end{array}$ & $\begin{array}{l}\text { Depth } \\
\text { (in.) } \\
\end{array}$ \\
\hline Any & Any & 0.003 or less \\
\hline $\begin{array}{l}100 \text { per } 15 \mathrm{sq} . \\
\text { in., randomly } \\
\text { distributed }\end{array}$ & $\begin{array}{l}\text { Less than } \\
0.030\end{array}$ & $\begin{array}{l}\text { Greater than } \\
0.003 \text { to } 0.017\end{array}$ \\
\hline 20 & $\begin{array}{l}0.030 \text { to } \\
0.050\end{array}$ & $\begin{array}{l}\text { Greater than } \\
0.003 \text { to } 0.017\end{array}$ \\
\hline
\end{tabular}

\section{RF Option B -}

Voids - There shall be no detectable voids. For limit of void detectability, see specification.

High Density Stringers - No high-density stringers with a Be equivalency greater than 0.020 inch shall be acceptable. No high-density stringers longer than 1.000 inch shall be acceptable. Not applicable to RM251520 and ME-001162.

Miscellaneous Inclusions - Inclusions with an absorption to $\mathrm{X}$ rays greater than that caused by a $10 \%$ increase in material thickness shall be limited as follows:

a. None with an effective size greater than 0.050 inch.

b. One per part with an effective size in the range of 0.030 to 0.050 inch inclusive.

c. In the range of 0.010 to $0.030 \mathrm{inch}$, there may be as many in any 1.0-inch-diameter circle projected on the radiograph as tabulated below:
Localized Thickness
(inches) 0 to 0.4

0.4 to 0.8

0.8 to 1.5

1.5 to 2.0

2.0 to 2.5

2.5 to 3.0

3.0 to 4.0

4.0 to 5.0

5.0 to 6.0
Max. No. of Inclusions Per Circle

3

8

12

23

30

37

47

60

73

\section{Surface Quality}

When examined visually, parts shall be free of detectable surface defects such as cracks, porosity, and scale.

\section{Radiograph Inspection}

Voids - There shall be no detectable voids. For limit of void detectability, see specification.

Inclusions - Inclusions with an absorption to $\mathrm{X}$ rays greater than that caused by a 10 percent increase in material thickness shall be limited as indicated below. Three inclusions in the range of 0.005 to less than $0.010 \mathrm{inch}$, which are included in any 0.125 -inch-diameter circle, shall be treated as one 0.010 -inch inclusion.

a. None with an effective size greater than 0.050 inch.

b. One per part with an effective size in the range of 0.030 to 0.050 inch inclusive.

c. In the range of 0.010 to $0.030 \mathrm{inch}$, there may be as many in any 1.0-inch-diameter 
TABLE 1. cont.

circle projected on the radiograph as

tabulated below.

\section{Local Part}

Thickness

(Inches)

0 to 0.4

0.4 to 0.8

0.8 to 1.5

1.5 to 2.0

2.0 to 2.5

2.5 to 3.0

3.0 to 4.0

4.0 to 5.0

5.0 to 6.0
Max. No. of Inclusions Per Circle

\section{3}

6

11

15

18

22

30

37

45

\section{Surface}

When examined by dye penetrant methods, finished parts shall be entirely free of cracks or linear discontinuities.

\section{Radiographic Inspection}

Voids - There shall be no detectable voids or porosity.

Inclusions - Inclusions with an absorption to $X$ rays greater than that caused by a 0.010 -inch beryllium equivalency shall be limited as follows:

a. None with an effective size greater than 0.025 inch.

b. In any 1-inch-diameter circle, there shall be no more than 3 inclusions with an effective size in the range 0.010 to 0.025 inch.

High Density Stringers - High-density stringers are unacceptable if they exceed any of the following:

a. Length - 1 inch

b. Width -0.015 inch

c. Radiographic density variation equivalent to 0.015 -inch beryllium thickness.

\section{Surface}

There shall be no indication of cracks or linear discontinuities as indicated by any nondestructive testing means capable of detecting this type defect.

\section{Radiographic Inspection}

Any defect that penetrates the entire wall thickness shall be cause for rejection of the part.

High-density inclusions having an absorption to $X$ rays greater than that caused by a 0.005 -inchthick beryllium penetrameter shall be limited as follows:

a. Any single void or inclusion greater than 0.050 -inch average dimension in the part shall cause rejection of the part.

b. More than 20 voids or inclusions with average dimensions between 0.030 and 0.050 inch shall be cause for rejection of the part.

c. No part shall contain more than 100 detectable voids or inclusions between 0.010 - and 0.030 -inch average dimension, randomly distributed, per 15 square inches of surface area.

d. Differences in radiographic film densities caused by nonuniformity in the part, which are not separately discernible as individual defects, shall be judged as a single defective area. Bonded areas appearing as higher density equal to or less than 0.005 equivalent radiographic absorption are acceptable. 
TABLE 2. Beryllium Powder

\begin{tabular}{|c|c|c|c|c|}
\hline SPEC/GRADE & $\mathrm{SP}-200-\mathrm{C}$ & P-20 & SP-100-C & $P-10$ \\
\hline AUTHORITY & Brush & KBI & Brush & $\mathrm{KBI}$ \\
\hline \multicolumn{5}{|c|}{ Chem. Comp. (\%) } \\
\hline $\mathrm{Be} \quad(\min )$ & 98 & 98 & 98.5 & 98.5 \\
\hline $\mathrm{BeO}$ (max) & 2.0 & 2.0 & 1.2 & 1.0 \\
\hline Al $(\max )$ & 0.16 & 0.15 & 0.14 & 0.08 \\
\hline B $\quad(\max )$ & -- & --- & $\ldots$ & -- \\
\hline $\mathrm{Cd}(\max )$ & $-\ldots$ & -- & -- & --- \\
\hline $\mathrm{Ca}(\max )$ & --- & --- & $-\ldots$ & $-\infty$ \\
\hline C $\quad(\max )$ & 0.15 & 0.15 & 0.15 & 0.10 \\
\hline $\mathrm{Cr} \quad(\max )$ & --- & -- & --- & --- \\
\hline Co $(\max )$ & $-\ldots$ & $--\infty$ & $\ldots-$ & $-\ldots$ \\
\hline $\mathrm{Cu} \quad(\max )$ & --- & -- & --- & -- \\
\hline $\mathrm{Fe} \quad(\max )$ & 0.18 & 0.18 & 0.15 & 0.15 \\
\hline $\mathrm{Pb} \quad(\max )$ & -- & $-\cdots$ & $-\ldots$ & -- \\
\hline $\mathrm{Li} \quad(\max )$ & $-\ldots$ & $\ldots$ & --- & --- \\
\hline $\operatorname{Mg}(\max )$ & 0.08 & 0.08 & 0.08 & 0.08 \\
\hline $\operatorname{Mn}(\max )$ & -- & --- & --- & --- \\
\hline Mo $(\max )$ & -- & $-m$ & --- & --- \\
\hline $\mathrm{Ni}(\max )$ & -- & $-\ldots$ & $-\infty$ & $-\ldots$ \\
\hline $\mathrm{N} \quad(\max )$ & --- & -- & $-\infty$ & $-\infty$ \\
\hline Si $(\max )$ & 0.08 & 0.08 & 0.08 & 0.08 \\
\hline $\mathrm{Ag} \quad(\max )$ & $-\ldots$ & -- & --- & --- \\
\hline Other : & 0.04 & 0.04 & 0.04 & 0.04 \\
\hline Partial & $\begin{array}{l}-200 \text { mesh } 75 \% \\
-100 \text { mesh } 98 \%\end{array}$ & $\begin{array}{l}-74 \text { micron } \\
(-200 \text { mesh })\end{array}$ & -200 mesh & $\begin{array}{l}-74 \text { microns } \\
(-200 \text { mesh })\end{array}$ \\
\hline Fabrication & -- & Impact Attrition & -- & Impact Attrition \\
\hline Remarks & $-\ldots$ & $\ldots-$ & -- & $\quad \quad \quad-\quad-$ \\
\hline
\end{tabular}


TABLE 2. cont.

$\begin{array}{ll}\text { NP-50-B } & \text { P-8 } \\ \text { Brush } & \text { KBI } \\ & \\ 99.0 & 99.0 \\ 0.9 & 0.80 \\ 0.075 & 0.07 \\ 0.00020 & 0.0002 \\ 0.0002 & 0.0002 \\ 0.01 & 0.01 \\ 0.10 & 0.10 \\ 0.01 & 0.01 \\ 0.0005 & 0.0005 \\ 0.015 & 0.015 \\ 0.075 & 0.085 \\ 0.002 & 0.002 \\ 0.0003 & 0.0003 \\ 0.05 & 0.08 \\ 0.012 & 0.015 \\ 0.002 & 0.002 \\ 0.02 & 0.02 \\ 0.02 & 0.02 \\ 0.06 & 0.06 \\ 0.0010 & 0.0010 \\ --- & --- \\ & \\ -74 \text { microns } & -74 \text { microns } \\ (-200 \text { mesh) } & (-200 \text { mesh }) \\ & \\ --- & \text { Impact Attrition } \\ --- & \\ & \end{array}$

P-1

KBI

99.0

0.8

0.01

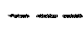

$-\cdots$

$\overline{0.03}$

0.0025

0.0005

0.0050

0.03

0.0005

$0 . \overline{06}$

0.003

0.001

0.02

0.01

$--$

$--$

-44 microns

( -325 mesh)

Impact Attrition

Electrolytic Flake Source
EF-1

KBI

99.0

0.5

0.0075

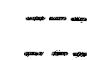

$-\infty-\infty$

0.0175

0.001

0.0005

0.003

0.01

0.0005

$\overline{0.003}$

0.001

0.001

0.006

0.0075

0.0005

C1 0.09

Zn-0.01

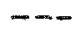

Electroly tic Flake Source 
RFP-2664 